\title{
Vivienda, un asunto de sustentabilidad urbana en México
}

\author{
Housing, an issue of urban sustainability in Mexico
}

\section{Salvador García-Espinosa}

Universidad Michoacana de San Nicolás de Hidalgo, Morelia (México)

Facultad de Arquitectura- Facultad de Economía

\section{María de la Luz Zavala-Villagómez}

Universidad Michoacana de San Nicolás de Hidalgo. Morelia (México)

Facultad de Economía

García-Espinosa , S., \& Zavala-Villagómez, M. de la L. (202I). Vivienda, un asunto de sustentabilidad urbana en México. Revista de Arquitectura (Bogotá), 23(2), 106-II5. https://doi.org/10.14718/RevArq.2021.3474

\author{
Salvador García-Espinosa \\ Arquitecto, Master en Planificación y Desarrollo Urbano, Universidad \\ Autónoma de México, México. \\ Master en Arquitectura, Investigación y Restauración de Sitios y Monu- \\ mentos, Universidad Autónoma de México, México. \\ Doctor en Geografía, Universidad Autónoma de México, México. \\ Profesor titular de tiempo completo en la Universidad Michoacana de \\ San Nicolás de Hidalgo. Las líneas de investigación son: Sustentabilidad \\ Urbana, Vivienda de Interés Social y Estructura Urbana https://scho- \\ lar.google.es/scholar?hl=es\&as sdt =0\%2C5\&q=Salvador + Garc\% \\ C3\%ADa + Espinosa\&oq = salva \\ (i)https://orcid.org/0000-0002-787I-5837 \\ (9) salvador.garcia.espinosa@umich.mx / salgaes I@gmail.com \\ María de la Luz Zavala-Villagómez \\ Arquitecta, Universidad Michoacana de San Nicolás de Hidalgo, México. \\ Doctora en Desarrollo Sustentable, Universidad Michoacana de San \\ Nicolás de Hidalgo, México. \\ Profesora en la Facultad de Arquitectura en la Universidad Michoacana \\ de San Nicolás de Hidalgo, México. \\ (D) https://orcid.org/0000-0002-3746-2730 \\ @arq_zv@hotmail.com
}

\section{Resumen}

En México, el $70 \%$ de sus habitantes vive en una ciudad y la expansión de la mancha urbana se presenta mayoritariamente a través del uso habitacional; de ahí la importancia de presentar una revisión de las políticas de vivienda gubernamentales; particularmente, la denominada como de interés social —es decir, aquella que se edifica a partir de la intervención del Gobierno en el otorgamiento de financiamientos-. El objetivo es evidenciar que se han privilegiado aspectos inherentes al uso eficiente de energía, a través de incorporar elementos de ecotecnias y relegando a un segundo término aspectos de índole urbana como la movilidad, los servicios y los equipamientos, razón por la que resulta impostergable el fortalecimiento de la política habitacional con indicadores de índole urbana que permitan crear una sinergia en la que la edificación de vivienda contribuya a la sustentabilidad urbana de las ciudades mexicanas. SINERGIA

Palabras clave: ciudad; expansión urbana; planificación urbana; urbanismo sustentable; vivienda

\section{Abstract}

In Mexico, $70 \%$ of its inhabitants live in a city, and the expansion of the urban sprawl is mainly due to housing use. Hence, the importance of reviewing government housing policies, particularly the so-called social interest housing, meaning, built through government intervention in the granting of financing. The objective is to show that factors inherent to the efficient use of energy have been privileged through the incorporation of eco-technologies, and relegated to a second-place urban aspect such as mobility, services and equipment. For this reason, it is urgent to strengthen housing policy with urban indicators that allow the creation of a synergy in which housing construction contributes to the urban sustainability of Mexican cities.

Keywords: city; housing; public policy; sustainable urbanism; urban planning; urban sprawl
Evaluado: marzo 23 / 2021

Aceptado: junio $30 / 2021$

\section{Introducción}

Este artículo sitúa la inherente relación que existe entre vivienda y sustentabilidad urbana. Se asume que la mancha urbana de cualquier ciudad se compone mayoritariamente del uso habitacional en sus diferentes modalidades. Se hace énfasis en lo relativo a la denominada vivienda de interés social, que conforma extensos conjuntos habitacionales, gracias al otorgamiento de créditos financieros por parte del Gobierno mexicano; sin embargo, no se respetaron los ordenamientos en materia urbana, y la edificación de millones de viviendas trajo consigo un impacto significativo al aumentar la dispersión de la mancha urbana, con sus consecuentes problemas en el funcionamiento, la administración y las condiciones ambientales de las ciudades mexicanas.

En México, la Constitución Política le otorga al gobierno la responsabilidad de proveer de vivienda a los trabajadores, razón por la cual se han desarrollado históricamente mecanismos de capitalización para el otorgamiento de créditos tendientes a la adquisición de vivienda para los trabajadores afiliados a distintos organismos de 
vivienda. Incluso, con la aprobación de la Ley de Asentamientos Humanos, en 1976, la política de vivienda se convirtió en un instrumento del gobierno para orientar el desarrollo urbano con la creación de extensas reservas territoriales en las cuales se edificaban miles de viviendas, además de obras de infraestructura y equipamiento.

Hacia finales de la década de 1990, se presentó un cambio radical en materia de vivienda: el gobierno dejó de ser el principal constructor de casas, para ceder estas actividades a promotores privados y reducir su participación al otorgamiento de créditos. Todo esto, bajo la premisa de que la oferta y la demanda regularían el mercado de la vivienda, situación que no ocurrió, pues la garantía del otorgamiento del crédito incentivó la especulación inmobiliaria, que se materializó en la edificación de millones de viviendas cuyo emplazamiento estuvo determinado por la búsqueda de suelo barato en las periferias de las ciudades. Y hoy, el crecimiento anárquico resulta ser una de las principales características de las ciudades mexicanas (Garza \& Schteingart, 2010, p. 84).

Con la finalidad de dimensionar el incremento en el número de viviendas que propició el cambio en la política habitacional, basta con señalar que, entre los diversos organismos creados para el financiamiento y la edificación de la vivienda, el de mayor importancia por el número de trabajadores afiliados es el Instituto del Fondo Nacional de la Vivienda para los Trabajadores (Infonavit), mismo que, desde su creación 1972 y hasta 2000, otorgó 2083429 créditos para vivienda, mientras que entre 2001 y 2006 se concedió un total de 1844510 créditos y en el sexenio de Felipe Calderón Hinojosa (2007-2012) se edificaron 6,6 millones de viviendas (Correa, 2013). Hoy en día, se sostiene la hipótesis de que la política de vivienda seguida en México se desarrolló más como parte de la estrategia económica para superar la crisis financiera que como parte de la estrategia social tendiente a disminuir déficits en materia de vivienda. El dato más evidente de esta realidad, es que para finales de 2019 se cuantificaron 5000000 de viviendas deshabitadas en el país.

Bajo la realidad planteada, el presente artículo tiene como objetivo evidenciar que la política de vivienda desarrollada en las últimas tres décadas generó impactos no deseados en términos de la movilidad urbana y la prestación de servicios básicos, entre otros muchos factores que, en conjunto, han impactado negativamente las posibles acciones tendientes a la sustentabilidad de las ciudades; además, señalar que las estrategias de sustentabilidad desarrolladas en torno a la vivienda se han centrado en aspectos de ahorro de energía y agua, cuyo impacto resulta mínimo ante los generados en el ámbito urbano.

\section{Metodología}

\section{Sustentabilidad en la vivienda}

Con el objetivo de mostrar cómo se han buscado impulsar acciones tendientes a lograr una sustentabilidad de la vivienda en México, se presenta a continuación un análisis sobre los principales programas de financiamiento gubernamental. Su presentación en orden cronológico tiene como objetivo proporcionar información sobre la evolución del concepto de sustentabilidad, pues, como se observará, con el paso del tiempo se van ampliando los elementos por considerar, hasta llegar recientemente a incorporar algunos del ámbito urbano.

Si bien los cambios en la política de vivienda, que incentivaron la edificación masiva de conjuntos habitacionales desde una perspectiva más económica que social, pueden ubicarse a finales de la década de 1990, será hasta 2006 cuando se comienza a manifestar una preocupación por la sustentabilidad y se realiza una modificación a la Ley de Vivienda, a fin de establecer que se deben "Priorizar las acciones de vivienda que constituya un factor de sustentabilidad ambiental, ordenación territorial y desarrollo urbano" (Ley de Vivienda, 2006, p. 4).

Se considera que dicha iniciativa en materia de vivienda evidencia la separación existente con las políticas urbanas, y de suelo, específicamente, de forma tal que las estrategias y las acciones para lograr la sustentabilidad de la vivienda se limitaron a integrar elementos tendientes a la eficiencia energética (Ley de Vivienda, 2017).

\section{Código de Edificación de Vivienda}

Tan solo un año después de la reforma en materia de la Ley de Vivienda, en 2007, la Comisión Nacional de Vivienda (Conavi) creó el Código de Edificación de Vivienda (Conavi, 2007), con la finalidad de unificar criterios y estandarizar las prácticas constructivas en todo el país. Comprende una compilación de una serie de requerimientos para la planeación, el desarrollo y la ejecución de diferentes edificaciones, así como una serie de parámetros de desarrollo urbano y arquitectónico que pretenden garantizar la habitabilidad y la sostenibilidad en las viviendas (Conavi, 2007, p. 302). Los elementos más relevantes que se contemplan son:

- Selección del sitio: Debe ser tal que se mitigue y se minimice el impacto ambiental y no se exponga la seguridad de la edificación ni la de sus habitantes. Seleccionar preferentemente suelos subutilizados dentro de la mancha urbana.

- Diseño y desarrollo del sitio: Busca que los recursos naturales se conserven al máximo, mediante un diseño que respete la vegetación existente y evite la erosión del suelo.

- Materiales de construcción: Que cumplan con las características de: no contener contaminantes; ser resistentes y que puedan ser separados con medios locales; ser renovables y abundantes; que impacten un mínimo el medio natural; no producir radiaciones naturales o inducidas; tener cualidades térmicas y acústicas; generar poco desperdicio, y ser reciclables.

- Uso eficiente de energía: Diseño bioclimático y aprovechamiento de energías renovables. Este apartado describe los sistemas de 
calefacción, aire acondicionado, envolventes térmicas y calentadores de agua de diversos tipos, entre otros aspectos más.

- Considerar la energía renovable como la primera condición para que la vivienda tenga el concepto de sustentable: Especialmente, la energía solar de forma pasiva (diseño bioclimático) o con dispositivos de conversión energética.

- Uso eficiente del agua: Parte de un abastecimiento con las características necesarias para el uso y el consumo humanos debe cumplir con las normas de hermeticidad de la red, cualidades de la toma domiciliaria y su respectivo medidor, así como una instalación hidrosanitaria adecuada, en la que se incluyan muebles y tuberías que cumplan con la normativa vigente.

- Aguas residuales: Estas deben ser colectadas mediante redes de alcantarillado, de forma que no se filtren al subsuelo; las descargas domiciliarias, el tratamiento de aguas y su reutilización deben ser de tal forma que no impliquen riesgos para la salud.

- Manejo adecuado de residuos sólidos: Debe caracterizarse por cumplir especificaciones de manejo y clasificación para optimizar su control, fomentar su aprovechamiento y minimizar su disposición final inadecuada.

- Áreas verdes: Deben realizarse mediante proyectos que, además, incluyan un programa de mantenimiento, criterios de sustentabilidad y educación ambiental y uso de especies nativas; además, deben tener un mínimo del $70 \%$ de cubierta vegetal, plantación de árboles y arbustos debajo del cableado eléctrico y en aceras o camellones, y disponer de riego (Conavi, 2010a, pp. 302-331).

\section{Programa Hipoteca Verde}

También en 2007, el Infonavit, principal organismo de financiamiento gubernamental de vivienda, puso en marcha el programa Hipoteca Verde, que representó un incremento en el monto del crédito para la adquisición de vivienda, con la finalidad de incorporar ecotecnias que permitieran la eficiencia energética y ambiental de esta. La lógica de operación consiste en considerar que el incremento en el costo inicial de la vivienda se verá compensado al disminuir la facturación por conceptos de energía y agua, de forma tal que este ahorro permite compensar el incremento del crédito hipotecario (Instituto del Fondo Nacional de la Vivienda para los Trabajadores [Infonavit], 2020).

Bajo el principio descrito, desde 2011 los créditos que otorga el Infonavit tienen la obligación de incorporar ecotecnias en las viviendas (Infonavit, 2020), conforme a las siguientes consideraciones:

a) Se debe incorporar una combinación flexible de ecotecnias, cuya eficiencia sea certificada.

b) Las ecotecnias pueden ser elegidas por el derechohabiente y validadas por las dependencias de Administración Pública Federal.

c) Las ecotecnias deben garantizar un ahorro mínimo progresivo, ligado al ingreso del trabajador.
El usuario final debe recibir una capacitación básica sobre el uso y el mantenimiento de las ecotecnias instaladas, así como las garantías con fecha de vigencia, dirección y teléfono de atención y servicio, para hacerlas efectivas, al igual que un manual de mantenimiento preventivo y correctivo impreso y, si es posible, electrónico (Infonavit, 2020, p. 11).

Este programa ha logrado un avance significativo en el uso más eficiente de la energía y del agua, así como la reducción del impacto ambiental debido a estos ahorros, pues se trata de casi 3000000 de viviendas edificadas hasta 2019, bajo los criterios descritos.

\section{Programa de certificación de edificaciones sustentables}

En 2008, tan solo un año después de la creación del Código de Edificación de Vivienda y de la puesta en marcha del programa Hipoteca Verde, se publicó en la Gaceta Oficial del Distrito Federal el Programa de Certificación de Edificaciones Sustentables (PCES), por parte de la Secretaría del Medio Ambiente (Sedema), que constituye un instrumento de planeación de política ambiental basado en las prácticas internacionales de certificación de sustentabilidad en las edificaciones (Sedema, 2008, p. 1).

Aunque el alcance de este último programa se limita a la Ciudad de México, capital del país, se lo incluyó en el análisis, por considerarse que representa el precedente de los códigos y los reglamentos para el resto del país (Sedema, 2008, p. 2). Los beneficios de la certificación de eficiencia traen consigo beneficios o incentivos para los propietarios de la vivienda, como la reducción en el pago de impuesto predial, la simplificación administrativa y posibles financiamientos a tasas preferenciales, así como a reducciones en primas de seguros, y beneficios que se encuentran en proceso de gestión, como el pago de cuotas preferenciales en los derechos del agua (Sedema, 2008, pp. 5-6). Los criterios de certificación contemplados son:

a) Energía: Se basa en el ahorro de energía (hasta 18 puntos) y uso de calentadores solares (7 puntos); los puntajes varían de acuerdo con la tipología de la edificación.

b) Agua: Privilegia la captación y la infiltración de aguas pluviales (hasta 5 puntos), el tratamiento y el uso de aguas grises (hasta 8 puntos) y el ahorro de agua potable (hasta 12 puntos).

c) Calidad de vida y responsabilidad social: Dependen de la tipología del edificio. Este rubro puede lograr un total de 25 puntos. Comprende: naturación de azotea, diseño bioclimático, acústica, mantenimiento, cultura de participación, abstención del uso de bienes de dominio público, y facilidad de transporte público. Un puntaje adicional optativo es otorgado por proveer áreas verdes para proporcionar confort y propiciar la interacción social (3 puntos), biciestaciones de préstamo de bicicletas (4 puntos), remodelaciones bioclimáticas (5 puntos) y ciclovía interna (4 puntos). 
d) Impacto ambiental y otros impactos: Incrementar los cajones de estacionamiento sin sacrificio de área libre (6 puntos); reciclaje de predios y respeto al uso del suelo $(1,5$ puntos cada uno); utilizar materiales locales, distancia reducida de proveedor, productos biodegradables, y materiales ambientalmente amigables (un punto cada uno), y respeto del arbolado existente (2 puntos).

e) Manejo de residuos: Almacenamiento temporal de residuos sólidos (3 puntos); señalamientos apropiados ( 0,5 puntos); mobiliario (1,5 puntos); separación de residuos (2 puntos); disposición final adecuada (3 puntos), y programa de sensibilización y difusión en materia de separación de residuos (un punto).

Como se ha podido observar, los diferentes programas analizados centran su atención en la vivienda y se limitan, fundamentalmente, a incentivar el ahorro en el consumo de energía eléctrica y agua potable, aspectos sin duda relevantes, pues de acuerdo con el Instituto Nacional de Estadística, Geografía e Informática (INEGI, 2018), las viviendas en México consumen el 16,23\% de la energía del país, el $65 \%$ del gas licuado, el $23 \%$ de la electricidad, el $100 \%$ de la leña y el $11,5 \%$ del agua, y emiten el $4,5 \%$ de los gases de efecto inverna- dero (GEI). Si se consideran los sectores residencial, comercial y público, el consumo se eleva al $19,6 \%$ de la energía nacional, lo que da el porqué del marcado énfasis en los aspectos señalados.

Resulta relevante señalar que al finalizar la primera década del siglo XXI se comenzaron a observar los impactos negativos propiciados por la política de edificación en materia de vivienda. Para 2012, el Inventario de Suelo alertaba que en las 129 ciudades con más de 50000 habitantes contabilizadas en México, existían más de 495000 hectáreas de suelo susceptible de ser incorporado al desarrollo urbano; también, 182000 hectáreas con uso habitacional. De estas últimas, el $17 \%$ son intraurbanas, y el $54 \%$, periurbanas (Conavi, 2010b, p. 5), lo que, sin duda, habla de la intensa dispersión urbana provocada.

$\mathrm{Al}$ parecer, el escenario descrito - y seguramente, su problemática en los ámbitos municipal y urbano- generó la necesidad de buscar reorientar la política de vivienda que se había seguido hasta 2010 e incluir la consideración de aspectos de índole urbana relacionados con el entorno de los conjuntos habitacionales. Muestra de este cambio, es que a partir de 2011 se lanza el programa Vivienda Sustentable, Vida Integral, del que se hablará a continuación.
Tabla 2. Principales características de los organismos certificadores nacionales.

Fuente: elaboración propia (2019).

\section{Entorno próspero}

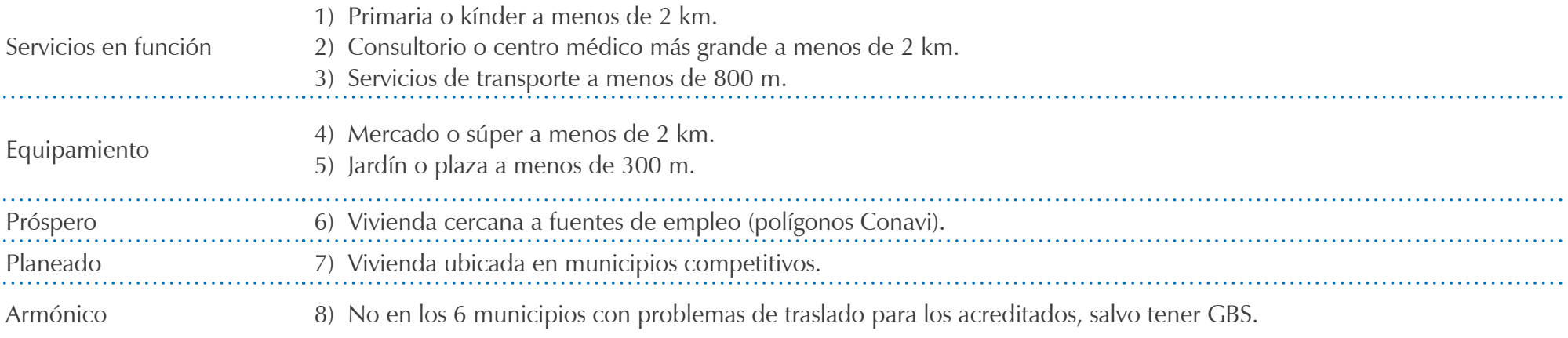

\section{Vivienda de calidad}

\begin{tabular}{|c|c|}
\hline Pavimentos y alumbrado & $\begin{array}{l}\text { 10) Banquetas. } \\
\text { 11) Alumbrado público. }\end{array}$ \\
\hline Conjunto compacto & 12) En área con densidad de vivienda mayor que 50 por hectárea. \\
\hline Cómoda & 13) Superficie total habitada mayor que $38 \mathrm{~m}^{2}$. \\
\hline Verde & 14) Hipoteca verde (vivienda ecológica). \\
\hline Digital & 15) Con acceso a internet. \\
\hline
\end{tabular}

\section{Comunidad solidaria y responsable}

Espacio comunitario

Predial mantenimiento

Promotor vecinal

Taller
16) A menos de $2 \mathrm{~km}$, centro comunitario para fraccionamientos menores de 1250 viviendas. O por lo menos, cancha deportiva para fraccionamientos menores de 350 viv.

17) Hipoteca con servicio: el acreditado autoriza al Infonavit a la retención de las cuotas de predial o a la conservación (la conservación podría realizarse también externamente).

18) Con promotor vecinal certificado y registrado en SRI.

19) Taller de inducción al derechohabiente: saber para decidir.

Notas: (SRI) Sistema de Redes de Infonavit, (GBS) Grado Básico de Sustentabilidad.

(A) Tabla 1. Mosaico de atributos de la vivienda sustentable.

Fuente: Infonavit (2012). 


\section{El programa Vivienda Sustentable, Vida Integral Infonavit}

Este programa afronta el reto de vincular los aspectos particulares de la vivienda relativos al uso energético eficiente, con el funcionamiento de la ciudad y el impacto que en términos de la movilidad se generan, así como con la demanda de servicios y equipamiento. Se fomenta el desarrollo de una vivienda sustentable, que incluya atributos de sustentabilidad desde tres ejes: ambiental, social y económico. Su objetivo es incentivar una vivienda que favorezca la responsabilidad de los vecinos con su entorno y su comunidad. Para esto, el programa contempla 20 atributos con el fin de proporcionar calidad de vida a los acreditados y salvaguardar su patrimonio (Infonavit, 2012, p. 2).

Entre las características solicitadas, destacan las agrupadas bajo el concepto de "Entorno próspero", y que refieren a la ubicación de la vivienda respecto al acceso a servicios públicos básicos (escuela o jardín de niños, servicio médico y transporte público), equipamiento urbano (área recreativa) y locales comerciales para abasto, como se muestra en la tabla 1.

De igual forma, en el ámbito normativo se publicó en 2013 la Norma Mexicana denominada Edificación sustentable, criterios y requerimientos ambientales mínimos, la cual se detalla a continuación.

\section{Edificación sustentable, criterios y requerimientos ambientales mínimos}

Identificada como NMX-AA-164-SCFI-2013, esta norma tiene como objetivo especificar los criterios y los requerimientos mínimos de una edificación sustentable (Norma Mexicana NMXAA-164-SCFI-2013, p. 8). Los parámetros de evaluación se determinan en los requisitos particulares, como se describe seguidamente:

1. Suelo: Se considera que la ubicación debe evaluarse en torno a la dotación de servicios e infraestructura y compatibilidad de uso del suelo, y no ubicarse en áreas naturales protegidas ni zonas de riesgo. También se toman en cuenta: regeneración urbana mediante el uso de inmuebles abandonados, porcentajes de áreas verdes mayor que el $10 \%$ y estacionamientos que no sumen área de desplante adicional a la de la edificación, entre otros (Norma Mexicana NMX-AA-164-SCFI-2013, pp. 27-32).

2. Energía: Demostrar una disminución de al menos el $10 \%$ en la ganancia de calor; cumplir con las normas de aislamiento térmico, y emplear al menos el $10 \%$ de la demanda de energía con energías renovables. Los calentadores de agua deben cumplir con la eficiencia térmica especificada; las lámparas, los refrigeradores y los congeladores deben cumplir con una eficiencia mayor, así como los acondicionadores de aire. Se establece un consumo máximo de energía expresado en $\mathrm{W} / \mathrm{m}^{2}$ considerado en el diseño; que no comprometan el confort ni la funcionalidad del edificio que depende de su tipología y de la zona bioclimática, y favorecer la iluminación natural (Norma Mexicana NMX-AA164-SCFI-2013, pp. 32-46).

3. Agua: Los materiales y los productos empleados deben ser certificados y reducir en al menos el $20 \%$ el consumo del agua. El uso de agua en pozos o a cielo abierto debe cumplir la normativa vigente, y puede captar agua de Iluvia y escurrimientos que reduzcan al menos el $25 \%$ de la descarga pluvial. Hasta el $30 \%$ de las aguas residuales se pueden enviar al alcantarillado público, y no descargar agua al arroyo de la calle. Si la edificación sobrepasa los $2500 \mathrm{~m}^{2}$, debe contar con una planta de tratamiento de aguas residuales y un sistema de tratamiento de lodos. Se puede aprovechar el gas de lodos, y el riego eficiente, preferentemente, en un horario que evite la evaporación. Diseño de pendientes y escurrimientos naturales, y cuidado adecuado de las aguas residuales durante la construcción (Norma Mexicana NMX-AA-164-SCFI-2013, pp. 46-49).

4. Materiales y residuos: Se deben considerar los impactos ambientales, sociales y económicos a lo largo del ciclo de vida de toda la edificación considerando obtención, manufactura, transporte, colocación, operación, mantenimiento, demolición y disposición final; también, cumplir con la normatividad vigente, y que los productos forestales acrediten su legal procedencia. Se pueden presentar análisis del ciclo de vida (ACV) comparativo de los materiales que se vayan a emplear; al menos el $50 \%$ del material puede ser reciclable, usar materiales con al menos el $10 \%$ de material reciclable, y reutilizar al menos el 30\% de la estructura en edificios reciclados (Norma Mexicana NMXAA-164-SCFI-2013, pp. 49-55).

5. Calidad ambiental y responsabilidad social: Cuidado de la biodiversidad; conservar la vegetación de más de $20 \mathrm{~cm}$ de diámetro y especies protegidas; divulgar información de especies nativas; proteger durante la construcción elementos naturales; integración del paisaje con áreas verdes y articulado con el sistema de espacios verdes de la ciudad (Norma Mexicana NMX-AA-164-SCFI-2013, pp. 55-58).

6. Paisaje: Los muros de colindancia y fachada pueden tener textura y color iguales a las de otras fachadas, o tener un recubrimiento vegetal; evaluar el impacto visual; las entradas deben dar preferencia al acceso peatonal y de vehículos no motorizados, y asegurar un diseño congruente con la topografía del terreno (Norma Mexicana NMX-AA-164-SCFI-2013, p. 58).

7 Calidad del ambiente interior: Deben existir parámetros de confort térmico con temperaturas entre $\operatorname{los} 18^{\circ} \mathrm{C}$ y $\operatorname{los} 25^{\circ} \mathrm{C}$, y favoreciendo las soluciones bioclimáticas sobre las mecánicas. Se debe tener una acústica promedio que no exceda los niveles sonoros (65 decibeles) y tiempos de exposición $(0,5$ segundos), que deben revisarse cada 3 años. 
Hacia el exterior, no debe producirse ruido que exceda los 70 decibeles acústicos (dBA) entre las 10 y las 22 horas (Norma Mexicana NMX-AA-164-SCFI-2013, pp. 59-61).

8. Responsabilidad social: Evitar barreras físicas que dificulten el acceso; contar con accesibilidad para personas con discapacidad y sectores poblacionales vulnerables; servicio de limpieza constante del edificio, y capacitación del personal y habitantes. En los sectores comercial, de servicios e industrial deben contar con un sistema de mejora continua respecto al confort y la habitabilidad del edificio; los usuarios deben contar con el espacio vital necesario para realizar sus actividades, y contar con cocina, comedor, baños y vestidores de empleados (Norma Mexicana NMX-AA164-SCFI-2013, pp. 61-62).

La norma también puntualiza la importancia de lograr un equilibrio entre los factores ambientales considerados a lo largo del ciclo de vida de la edificación, conforme a las condiciones locales imperantes y a los aspectos de localización, desempeño, aprovechamiento y diseño, y cumpliendo con una serie de disposiciones de carácter obligatorio.

Desde la misma perspectiva, con la participación de la fundación Implementación, Diseño y Evaluación de Políticas Públicas (IDEA) y una diversidad de organismos públicos, en 2013 se diseñó la Estrategia Nacional para la Vivienda Sustentable (ENVS), descrita a continuación.

\section{Estrategia Nacional para la Vivienda Sustentable}

Con el objeto de que México adopte prácticas similares a las del Reino Unido y establecer un sistema de calificación para la eficiencia energética y la vivienda verde en México, este proyecto busca la

Asistencia en el diseño, validación e implementación de un esfuerzo multi-agencial para promover el crecimiento verde en el mercado hipotecario en México por medio del incremento de la eficiencia energética de unidades habitacionales con el uso de una base metodológica unificada. (IDEA, 2013, p. 12)

El Sistema de Evaluación de Vivienda Verde INFONAVIT (Sisevive) cuenta con dos herramientas de cálculo: el desempeño energético de la vivienda (DEEVi) (basada en Passive House Institute [PHPP], y adaptada a las condiciones mexicanas), para evaluar el desempeño energético, y el Simulador de Ahorro de Agua en la Vivienda (SAAVi), para evaluar el consumo de agua en una vivienda. Se toman en cuenta tres aspectos para la calificación final: la demanda específica total (refrigeración y calefacción), la demanda de energía primaria (electricidad y gas) y el consumo proyectado de agua (Fundación IDEA, 2013, p. 13).

Adicionalmente, en 2011, en el marco del Programa México-Alemán para Acciones de Mitigación Nacionalmente Apropiadas (conocido como NAMA), el Ministerio Federal de Medio Ambiente, Protección de la Naturaleza y Seguridad Nuclear, así como la Sociedad Alemana para la Cooperación Internacional (conocida como GIZ, por sus siglas en alemán) asesoraron a la Conavi para desarrollar la Acción de Mitigación Nacionalmente Apropiada (NAMA) en el sector de vivienda social que se basa en el desempeño global de la vivienda considerando la eficiencia energética total, y se proponen tres diferentes estándares de desempeño: EcoCasa 1, EcoCasa 2 y EcoCasa Max, para las principales zonas bioclimáticas de México, con el objetivo de ampliar la penetración de estándares de eficiencia básicos (similares a Hipoteca Verde) a todo el mercado de la vivienda en nuestro país (Fundación IDEA, 2013, p. 13).

Así mismo, una acción encaminada a la sustentabilidad de las viviendas en nuestro país son las Reglas de Operación del Programa de Subsidios Federales Esta es tu casa, que desde 2009 incorporan requisitos para que las viviendas susceptibles de recibir subsidio tengan condiciones mínimas de sustentabilidad. En un inicio, el enfoque fue la existencia de ecotecnias en las viviendas, pero de forma gradual se ampliaron los objetivos a factores de ubicación, viviendas verticales y uso del agua (Fundación IDEA, 2013, p. 19).

La ENVS tiene una visión estratégica compartida, y reconoce como principal objetivo la calidad de vida de las familias, por lo que la vivienda como espacio de bienestar debe garantizar confort, higiene, alimentación, educación y entretenimiento a sus habitantes, al satisfacer las demandas de energía y agua para satisfacer las necesidades cotidianas buscando el mínimo impacto ambiental posible (Fundación IDEA, 2013).

Sin duda alguna, la problemática urbana que se detonó con la edificación indiscriminada de vivienda fue tal que incluso organizaciones de la sociedad y centros de investigación se sumaron a la elaboración de propuestas. Tal es el caso del Centro Mario Molina (CMM), que desarrolló una herramienta para diagnosticar el comportamiento ambiental, económico y social de la vivienda y su espacio urbano en México, denominado Índice de Sustentabilidad de la Vivienda y su Entorno (ISV), que mediante los 30 indicadores del ISV — Cuyo desempeño está condicionado a 40 factores de ponderación regional— pretende evaluar la sustentabilidad de la vivienda, a partir de fijar una escala de valor que determina el peso de las variables con base en las características de la región (Centro Mario Molina, 2012, pp. 1-3). Los tres ámbitos en los que se agrupan dichos indicadores son:

1. Índice ambiental: Contempla uso del suelo, abastecimiento de agua y energía, electricidad, formación de oxidantes fotoquímicos, cambio climático, toxicidad humana, acidificación, eutrofización, y manejo y disposición de residuos sólidos.

2. Índice económico: Considera variación del gasto familiar, ahorros por la implementación de ecotecnias, gastos de vivienda por porcentaje de ingresos, gastos de transporte como porcentaje del ingreso, formación de patrimonio y plusvalía. 
3. Índice social: Integran la variación, la adecuación y la influencia del tamaño de los espacios en las relaciones familiares, la calidad de los materiales, el índice de hacinamiento, las variaciones del entorno, la suficiencia de equipamiento urbano, la organización de los vecinos, los espacios públicos y la convivencia social, entre otros más (Centro Mario Molina, 2012).

Finalmente, los factores de ponderación para cada uno de los índices describen cómo establecer los criterios de acuerdo con la categoría de impacto para los indicadores considerados (Centro Mario Molina, 2012, p. 8).

\section{Resultados}

\section{La sustentabilidad de la vivienda}

Los programas de incorporación de ecotecnias en la vivienda representan un incremento en su costo, que puede llegar a ser de hasta del 30\%. Se asume que el propietario logrará amortizar este costo inicial con la disminución en la facturación de los consumos; principalmente, los de energía eléctrica y de agua potable. Este sobrecosto, sin embargo, representa un inconveniente para una construcción sustentable, dado que donde existe el mayor déficit cuantitativo y cualitativo de vivienda es, precisamente, en la población de menor poder adquisitivo.

Por el contrario, en programas como Vivienda Sustentable, Vida Integral Infonavit cabe hacer notar que los principales beneficios económicos se los lleva el desarrollador, pues cuando la vivienda califica como "vivienda sustentable" el Infonavit paga de inmediato el costo total de la vivienda, aun cuando el trabajador no haya iniciado el trámite del crédito; además, todas estas viviendas reciben financiamiento por parte del instituto desde la fase de construcción, y adicionalmente se puede tener acceso a otro tipo de estímulos fiscales y económicos (Infonavit, 2012, p. 11).

Existe en el diseño de los programas o los planes de vivienda una preocupación por su inserción en la ciudad, como en el programa Vivienda Sustentable, Vida Integral Infonavit, donde hay una categoría denominada "entorno próspero", que busca una clara complementariedad entre la vivienda y el entorno del cual esta forma parte; es decir, la prosperidad económica y social de sus habitantes dependerá de la integración y la consolidación del entorno urbano. En este sentido, hay que señalar que las distancias consideradas mínimas para garantizar el acceso a servicios y equipamientos básicos son fácilmente eludibles en la práctica; tal es el caso de los consultorios médicos, ya que su validación no considera si se trata de un módulo provisional o farmacia con consultorio, ni si corresponde al sistema de salud gubernamental o se trata de un servicio privado, al que difícilmente tienen acceso los habitantes de la vivienda.

Algo similar acontece con el equipamiento educativo básico de jardín de niños, pues el único requisito es que la institución se encuentre registrada ante la Secretaría de Educación Pública, sin importar si se trata de un colegio público o uno privado. En el caso del mercado, si bien se considera que está enfocado en el abasto de productos básicos alimenticios, bastará con que el desarrollo habitacional registre que cuenta con cinco locales comerciales para validar este rubro, sin que, necesariamente, su ocupación sea bajo el giro comercial de abasto.

Como ya se mencionó, el Programa de Certificación de Edificaciones Sustentables tiene un alcance limitado solo a la Ciudad de México; desafortuna-

Social Variación y adecuación de los espacios en las relaciones familiares, hacinamiento, equipamiento, organización vecinal y tiempo de transporte.

\begin{tabular}{|c|c|c|c|}
\hline \multirow[b]{2}{*}{ SEDEMA-PCES } & Ambiental & Energía, agua, impacto ambiental y otros impactos, y manejo de residuos. & \multirow{2}{*}{ Sí } \\
\hline & Social & $\begin{array}{l}\text { Calidad de vida y responsabilidad social, } \\
\text { confort de los espacios e integración social. }\end{array}$ & \\
\hline CONAVI-CEV & Ambiental & $\begin{array}{l}\text { Selección del sitio, conservación de recursos naturales, materiales de cons- } \\
\text { trucción, energía, energía renovable, uso eficiente del agua, manejo de aguas } \\
\text { residuales, manejo de residuos sólidos, y áreas verdes. }\end{array}$ & No \\
\hline \multirow{3}{*}{$\begin{array}{l}\text { NMX-AA-164- } \\
\text { SCFI-2013 }\end{array}$} & Ambiental & Suelo, energía, agua, materiales y residuos, y calidad ambiental. & \multirow{3}{*}{ Sí } \\
\hline & $\begin{array}{l}\text { Económico } \\
\ldots \ldots \ldots \ldots \ldots \ldots\end{array}$ & 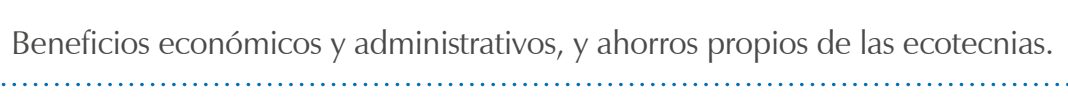 & \\
\hline & Social & Responsabilidad social y calidad del ambiente interior. & \\
\hline
\end{tabular}


damente, las certificaciones son voluntarias y los certificados de edificación sustentable, de acuerdo con el grado de cumplimiento de los criterios de sustentabilidad, tienen tres categorías: cumplimiento (de 21 a 50 puntos), eficiencia (de 51 a 80 puntos) y excelencia (de 81 a 100 puntos). Eso lleva a que el propietario valore el posible costo-beneficio entre el posible grado de certificación que obtendrá y los posibles beneficios en la reducción de tarifas de servicios básicos o el cobro de impuestos gubernamentales.

Dentro de la ENVS, el programa EcoCasa, operado por la Sociedad Hipotecaria Federal (SHF), destaca los Desarrollos Urbanos Integrales Sustentables (DUIS) y apoya acciones para que las viviendas alcancen mayores niveles de eficiencia y sustentabilidad, mediante acciones conjuntas de los sectores público y privado (Fundación IDEA 2013, p. 19). La propia Secretaría de Desarrollo Agrario, Urbano y Territorial (Sedatu) señaló que

Hoy 3 de cada 10 viviendas que subsidia el Gobierno de la República mitigan aproximadamente 25 por ciento de sus emisiones de Gases de Efecto Invernadero (GEI), y contemplan criterios de eficiencia energética e hídrica mitigando más de 54 mil toneladas de carbono al año equivalente a plantar un millón 800 mil árboles. (Sedatu, 2018, párr. 2)

Lo anterior, sin duda, viene a reafirmar la prioridad del enfoque en la vivienda en menoscabo del enfoque urbano y territorial.

\section{La vivienda en la sustentabilidad urbana}

Las ciudades son "los espacios del hábitat social donde se desarrolló la vida en comunidad, satisfacen sus necesidades, generan recursos, y cultura, es decir, es donde generan una expresión el desarrollo social y económico" (Jordán \& Simoni, 2003, p. 44); de ahí que hablar de la sustentabilidad urbana necesariamente remite al concepto de desarrollo sustentable, impulsado primordialmente por las Naciones Unidas, la Organización para la Cooperación y el Desarrollo Económico (OCDE) y el Banco Mundial, así como la Unión Europea, y que se comprende a partir de tres elementos básicos: la sustentabilidad ecológica o ambiental, la social y la económica.

Aunque la multidimensional del concepto de desarrollo sustentable tiende a caer en grandes generalizaciones, para el ámbito urbano se puede considerar que el proceso de planificación debe lograr la coincidencia entre los objetivos sociales, ambientales y económicos, que darán como consecuencia un desarrollo urbano sustentable. Se trata de operativizar el concepto; de ahí que resulta oportuno retomar como definición de ciudad sostenible la de

[...] un núcleo urbano en el que la utilización de los recursos naturales se produce de manera congruente con el objetivo de poder trasmitir a las generaciones futuras similares o mejores disponibilidades de los mismos, o de otros alternativos necesarios para funciones semejantes. (Zoido \& Lois, 2000, p. 92)

Lezama y Domínguez (2006, p. 160) indican que los componentes desarrollo y sustentabilidad, en su dimensión urbana, no pueden limitarse a garantizar o promover la permanencia del capital natural mínimo requerido para asegurar la continuidad de la especie humana. Deben también proveer los elementos necesarios para efectuar un acceso más equitativo, más igualitario y más democrático a la riqueza natural.

Lo anterior implica un cambio radical en la dirección que ha seguido el desarrollo de las ciudades en México, pues hoy en día, como ya se indicó, las caracteriza una dispersión de la mancha urbana, que es la expresión territorial de una concepción económica predominante. En otras palabras, la insustentabilidad se propicia al privilegiar la visión económica por sobre la social y la ambiental.

Además de lo anterior, existen impactos en el ámbito social. El caso más agudo es la movilidad, pues los tiempos de recorrido se incrementan a costa de una disminución del tiempo de convivencia, descanso o actividades culturales de los habitantes de esos desarrollos habitacionales, muchas veces calificados como ciudades dormitorio, pues sus moradores acuden allí prácticamente a dormir y a nada más, y están ausentes durante el día, con los diversos problemas sociales que esto implica.

Ahora bien, es deseable considerar la idea de la sustentabilidad urbana como un camino o una trayectoria, donde las diferentes acciones realizadas pueden contribuir a propiciar la coincidencia o el equilibrio entre los ámbitos ambiental, económico y social. En este sentido, se comprende que la búsqueda de la eficiencia energética de la vivienda — es decir, la visión ecoenergética de la viviendacontribuye en esta trayectoria.

También, sin embargo, adquiere relevancia la reflexión en torno a la vivienda, pero no solo en términos de la visión ecoenergética, sino en su calidad de componente urbano, donde su contribución a la sustentabilidad de la ciudad dependerá no tanto de sus características edificatorias, sino en mayor medida, de su emplazamiento en torno a la accesibilidad a equipamientos básicos de salud, educación y abasto, además de áreas verdes, entre otros muchos factores más.

De inicio, hay que señalar que la incidencia de las inversiones en vivienda, en virtud de que fueron desarrolladas por empresarios privados, buscaron concentrar la oferta de vivienda en las ciudades donde se concentraba el mayor número de trabajadores afiliados a los organismos de vivienda, y no necesariamente donde se presentaba el mayor déficit. En los últimos 20 años, más del $50 \%$ de las viviendas construidas se concentró en solo ocho de las 32 entidades federativas de México, donde destacan: Nuevo León, Estado de México, Jalisco, Ciudad de México, Chihuahua, Baja California, Guanajuato y Tamaulipas (ONU-Hábitat, 2018, p. 21). 
Para el caso específico de las ciudades mexicanas, se observan una estructura urbana y un patrón de dispersión que son resultado de la búsqueda de la máxima rentabilidad al urbanizar; solo así se explica el caso de los desarrollos habitacionales construidos en la periferia de las ciudades, bajo la lógica de buscar el suelo más barato. Una evidencia de lo anterior es que, de acuerdo con el Instituto Nacional de Suelo Sustentable, en su diagnóstico de la Política Nacional de Suelo, señala que entre 1980 y 2017 la superficie de sus ciudades se incrementó a un ritmo del 5,4\% cada año, mientras que la población urbana de México creció a una tasa promedio anual del 2,4\% (Sedatu, 2020, p. 43). Es decir, las ciudades crecieron en su extensión 2,3 veces más rápido que su población (ONU-Hábitat, 2018, p. 110).

La edificación de viviendas ha sido un factor clave en propiciar la dispersión: así lo demuestra el hecho de que el $85 \%$ de la vivienda financiada con un crédito Infonavit se ubica en las zonas intermedias y periféricas de los núcleos urbanos, mientras que el $10 \%$ está en zonas de expansión y el $5 \%$ se ubica en zonas céntricas.

Sin duda alguna, considerando que el uso habitacional representa el de mayor extensión en la mancha urbana de cualquier ciudad, puede afirmarse que los subsidios a préstamos hipotecarios para adquisición de vivienda han sido factor impulsor de la expansión, pues la oferta de viviendas nuevas se concentró en la periferia de las ciudades. Según datos del Registro Único de Vivienda (RUV), entre 2006 y 2013 más del 70\% de los hogares registrados en el nuevo registro de la vivienda fueron construidos ya sea en zonas de ubicación intermedia (U2) o en zonas periféricas (U3) (ONU-Habitat, 2018, p. 112).

Un factor que incide de forma determinante en la densidad es que aproximadamente el 90\% del parque habitacional está formado por casas individuales (INEGI, 2010). Entre 2000 y 2010, en las zonas metropolitanas con al menos 500000 habitantes, la zona centro-ciudad registró una caída promedio del $7,5 \%$ en la densidad de población; en contraste, la densidad de población en las zonas situadas a más de $10 \mathrm{~km}$ del centro de la ciudad aumentó en el 6,8\% en promedio (INEGI, 2000; 2010). Las ciudades mexicanas presentan un modelo de crecimiento disperso con una densidad promedio urbana cercana a las 23 viviendas por hectárea en 2015.

Dicha dispersión urbana no solo se traduce en una ineficiencia de la infraestructura instalada, sino que uno de los mayores impactos visibles se deriva de la movilidad, por el tiempo y el costo que invierten las familias en tal sentido. En las localidades con más de 2500 habitantes, para estos, el transporte es el segundo concepto de gasto familiar, con el $19,3 \%$, seguido de la educación, con el 12,4\%, lo que duplica el gasto en vivienda y sus servicios, con el 9,5\%, y es siete veces más que lo gastado en cuidados a la salud, con el 2,7\% (INEGI, 2016).

Finalmente, la evidencia más clara que sustenta la hipótesis de que la política habitacional se ha utilizado más como parte de una estrategia económica es el hecho de que a principios de 2019, las viviendas que se encontraban deshabitadas o que nunca fueron ocupadas se cuantificaron en 5000000 (Martínez, 2017), mientras que en el ámbito nacional, en las viviendas pertenecientes a los cuatro deciles inferiores de ingresos, el rezago de vivienda representa el 58\% (INEGI, 2015).

\section{Discusión}

Como se pudo observar en los diferentes documentos analizados, existe un marcado interés en los aspectos materiales con los que se edifica la vivienda; en segundo término se ubican los aspectos relacionados con el uso de ecotecnias o el uso de materiales que propicien una eficiencia térmica de la casa. En lo que se podría identificar como una segunda etapa, surge la preocupación por considerar aspectos relacionados con el suelo, el abastecimiento de agua, las áreas verdes y los residuos sólidos; es decir, la dimensión ambiental de la vivienda, así como un bloque que agrupa los aspectos sociales relacionados con el acceso a equipamientos básicos, hacinamiento y transporte, factores que, implícitamente, dependen del emplazamiento de la vivienda con respecto a la mancha urbana de la ciudad.

Hay que subrayar que solo en tres casos (Programa de Certificación de Edificación Sustentable, Código de Edificación de la Vivienda y Norma Oficial Mexicana) se integran aspectos urbanos tales como el emplazamiento de la vivienda, la dotación de servicios e infraestructura y el uso del suelo; incluso, se llega a señalar que una vivienda sustentable es aquella en la que coinciden de forma exitosa indicadores de dos ámbitos distintos: los de naturaleza urbana (acceso y cercanía al equipamiento básico de educación, salud y abasto) y los relacionados con la calidad de la vivienda (hipotecas verdes, disponibilidad de vialidades pavimentadas y una densidad mínima de 50 viviendas/hectáreas).

\section{Conclusiones}

La participación de la iniciativa privada en la construcción de la vivienda ha sido un factor clave para que los promotores, en su búsqueda permanente de suelo barato, edifiquen desarrollos habitacionales cada vez más alejados de la mancha urbana, e incentiven así la dispersión urbana (SEDESOL, 2010, p. 8). Un análisis sobre conjuntos habitacionales construidos de 1996 a 2006 identificó que el $30 \%$ de los desarrollos habitacionales tienen como vía de acceso una vialidad regional. Incluso la distancia promedio de estos desarrollos respecto al centro urbano se ha ido incrementando con el paso de los años, al ser de $21,9 \mathrm{~km}$ en las megalópolis; de 12,69 km, en las ciudades grandes, y de 6,16 km, en las medianas (Roberto \& Goya, 2009).

En tres décadas, la superficie urbana en México creció a un ritmo de 50 hectáreas diarias; la población se duplicó en ese periodo, mientras que la superficie 
urbanizada se multiplicó por diez (SEDESOL, 2010, p. 10). Las manifestaciones de dicha dispersión se dejan ver en la dimensión social, la ambiental y la económica; ejemplo de esto es el costo directo del transporte, el tiempo que deben invertir en traslado (Pulido et al., 2017) y la contaminación por el incremento de vehículos automotores, además de considerar que la dispersión urbana incrementa los costos en el funcionamiento de la ciudad. En otras palabras, el promotor de vivienda, traslada hacia la ciudad y sus habitantes sus externalidades.

Se considera que pretender actuar a través de los mecanismos de financiamiento a la vivienda genera un alcance limitado, razón por la que no ha generado los efectos esperados en las ciudades mexicanas. Para lograr impactos relevantes, se hace necesario actuar desde la perspectiva de la planificación y la administración urbanas, a fin de evitar la autorización de más desarrollos habitacionales alejados de la mancha urbana.

Considerar que existe una gran cantidad de viviendas deshabitadas, hace previsible que no se edificarán muchas viviendas en el futuro inmediato; sin embargo, la problemática generada que enfrentan, hoy en día, las ciudades mexicanas es en términos de su sustentabilidad, pues la dispersión urbana generada propició problemas de movilidad urbana, cobertura de servicios básicos, además del equipamiento urbano necesario para la población que habita en estos conjuntos habitacionales de la periferia urbana (Sobrino et al., 2018).

\section{Contribuciones y agradecimientos}

El presente artículo se deriva de una investigación de tesis doctoral desarrollada por la Dra. Zavala Villagómez, intitulada Sustentabilidad, Vivienda y Desarrollo Urbano, misma que se desarrolló bajo la dirección del Dr. García Espinosa, por corresponder a su línea de investigación desarrollada como profesor investigador titular de tiempo completo, dentro del Programa de Doctorado en Desarrollo y Sustentabilidad, que se imparte en la Facultad de Economía de la Universidad Michoacana de San Nicolás de Hidalgo, programa PNPC CONACYT.

\section{Referencias}

Centro Mario Molina. (2012). Evaluación de la sustentabilidad de la vivienda en México. https://centromariomolina.org/ ciudades-sustentables/evaluacion-de-lasustentabilidad-de-la-vivienda-en-mexico/

Comisión Nacional de Vivienda (Conavi). (2007). Código de edificación de vivienda. https://www.gob.mx/conavi/documentos/ codigo-de-edificacion-de-vivienda

Comisión Nacional de Vivienda (Conavi). (2010a). Código de vivienda. México D.F.

https://www.bibliocad.com/es/biblioteca/ codigo-de-vivienda-conavi_87445/

Comisión Nacional de Vivienda (Conavi). (2010b). Guía para la redensificación habitacional en la ciudad interior.

http://cdam.unsis.edu.mx/files/Desarrollo Urbano y Ordenamiento Territorial/Otras disposiciones/Guía para la Redensificación.pdf

Correa, G. (2013). Construcción y acceso a la vivienda en México; 2000-2012. Intersticios Sociales, 7, 31.

http://www.scielo.org.mx/scielo.php?script=sci arttext\&pid=S2007-49642014000100005

Garza, G. \& Schteingart, M. (2010). Desarrollo Urbano y Regional (Primera). Colegio de México.

https://2010.colmex.mx/16tomos/II.pdf

Fundación IDEA. (2013). Estrategia nacional para la vivienda sustentable. http://fundacionidea.org.mx/posts/51

Instituto Nacional de Estadística, Geografía e Informática (INEGI). (2000). Censo de Población y Vivienda.

Instituto Nacional de Estadística, Geografía e Informática (INEGI). (2010). Censo de Población y Vivienda.

Instituto Nacional de Estadística, Geografía e Informática (INEGI). (2015). Principales resultados de la Encuesta Intercensal 2015 Estados Unidos Mexicanos.

http://internet.contenidos.inegi.org. $\mathrm{mx} /$ contenidos/productos/prod_serv/ contenidos/espanol/bvinegi/productos/ nueva estruc/702825078966.pdf

Instituto Nacional de Estadística, Geografía e Informática (INEGI). (2016). Encuesta Nacional de Ingresos y Gastos de los Hogares 2016 (ENIGH).

https://www.inegi.org.mx/programas/enigh/ nc/2016/

Instituto Nacional de Estadística, Geografía e Informática (INEGI). (2018). Comunicado de prensa 541/18: Primera encuesta nacional sobre consumo de energéticos en viviendas particulares (ENCEVI).

https://www.inegi.org.mx/contenidos/ saladeprensa/boletines/2018/EstSociodemo/ ENCEVI2018.pdf

Instituto del Fondo Nacional de la Vivienda para los Trabajadores (Infonavit). (2012). Programas del Infonavit para desarrolladores.

https://portalmx.infonavit.org.mx/wps/ portal/infonavit.web/proveedores-externos/ para-tu-gestion/desarrolladores/programadesarrolladores/!ut/p/z1/jZDNCoJQEEafpYX LnFHjYuOOzBIpgySbTWjYTTCvqCW9fdLPIs hbs5vhnJnhA4llqlivGY-bTBRx3vU7Ynvmls6 nI23peqjjemVvglC3Dd_XY

Instituto del Fondo Nacional de la Vivienda para los Trabajadores (Infonavit). (2020). Manual explicativo de la vivienda ecológica 2020: Hipoteca verde. Infonavit.

Jordán, R., \& Simoni, D. (2003). Gestión urbana para el desarrollo sostenible en América Latina y el Caribe. CEPAL.

Ley de Vivienda, reformada, Diario Oficial de la Federación [D.O.F.], 27 de junio de 2006 (México).

http://www.diputados.gob.mx/LeyesBiblio/ pdf/LViv 140519.pdf

Ley de Vivieñda, reformada, Diario Oficial de la Federación [D.O.F.], 23 de junio de 2017 (México).

https://www.dof.gob.mx/nota_detalle. php? codigo $=5487938 \&$ fecha $=23 / 06 / 2017$

Lezama, J. L.. \& Domínguez, J. (2006). Medio ambiente y sustentabilidad urbana. Papeles de Población, 12(49), 153-176.

http://www.scielo.org.mx/scielo.php?script=sci arttext\&pid=S1405-74252006000300007

Martínez, A. N. (2017). Vivienda en abandono. Análisis socioespacial de la vivienda abandonada en México. Territorios en Formación, O(12), 41.

https://doi.org/10.20868/tf.2017.12.3647

Norma Mexicana NMX-AA-164-SCFI-2013 Edificación sustentable, criterios y requerimientos ambientales mínimos, Diario Oficial de la Federación [D.O.F.] 4 de septiembre de 2013 (México)

ONU-Habitat. (2018). Vivienda y ODS en MéxiCO. ONU.

Pulido, J. A. L., Díaz, G. E., Gómez, J. C. Z., \& Sanginés, A. G. (2017). Los costos de la expansión urbana: Aproximación a partir de un modelo de precios hedónicos en la zona metropolitana del valle de México. Estudios Demográficos y Urbanos 32. https://estudiosdemograficosyurbanos. colmex.mx/index.php/edu/article/ view/1615/1672

Roberto, E., \& Goya, C. (2009). Estudio de la integración urbana y social en la expansión reciente de las ciudades en México 19962006: dimensión, características y soluciones. SEDESOL, UAM, Porrúa, Ed. http://biblioteca.diputados.gob.mx/janium/ bv/ce/scpd/LX/estud_urb.pdf

Secretaría de Desarrollo Agrario, Urbano y Territorial (Sedatu). (2018). La estrategia nacional de vivienda sustentable, visión de largo plazo dirigida a la vivienda de interés social.

https://www.gob.mx/sedatu/prensa/ la-estrategia-nacional-de-vivienda-sustentablevision-de-largo-plazo-dirigida-a-la-vivienda-deinteres-social-sedatu-161568?idiom =es-MX

Secretaría de Desarrollo Agrario, Urbano y Territorial (Sedatu). (2020). Política Nacional de Suelo. Sedatu.

Secretaría del Medio Ambiente (Sedema). (2008). Programa de certificación de edificaciones sustentables.

https://www.sedema.cdmx.gob.mx/storage/ app/media/tramites-servicios/auditoriaautoregulacion-ambiental/edificacionessustentables/programa-certificacionedificaciones-sustentables.pdf

SEDESOL. (2010). Guía para la redensificación habitacional en la ciudad interior.

http://cdam.unsis.edu.mx/files/Desarrollo Urbano y Ordenamiento Territorial/Otras disposiciones/Guía para la Redensificación.pdf

Sobrino, J., Garrocho, C., Graizbord, B., Brambila, C., Adrián, \& Aguilar, G. (2018). Ciudades sostenibles en México: una propuesta conceptual y operativa. ONU.

Zoido, F. \& Lois, R. (2000). Diccionario de geografía urbana, urbanismo y ordenación del territorio. Ariel.

https://dialnet.unirioja.es/servlet/ libro? codigo $=766185$ 

(1) Portada: Las bovedadas de Bramante.

Fotografía: Luis Alberto Martínez Camacho (2021) CC BY-NC

\section{(ब) (1) (\$)}

(A) Orientación editorial

\section{Enfoque y alcance}

La Revista de Arquitectura (Bogotá) ( (ISSN 1657-0308 Impresa y E-ISSN 2357-626X en línea) es una publicación científica seriada de acceso abierto, arbitrada mediante revisión por pares (doble ciego) e indexada, en donde se publican resultados de investigación originales e inéditos.

Está dirigida a la comunidad académica y profesional de las áreas afines a la disciplina. Es editada por la Facultad de Diseño y el Centro de Investigaciones (CIFAR) de la Universidad Católica de Colombia en Bogotá (Colombia).

La principal área científica a la que se adscribe la Revista de Arquitectura (Bogotá) según la OCDE es:

Gran área: 6. Humanidades

Área: 6.D. Arte

Disciplina: 6D07. Arquitectura y Urbanismo

También se publican artículos de las disciplinas como 2A02, Ingeniería arquitectónica; 5C03, Estudios urbanos (planificación y desarrollo); 6D07, Diseño.

Los objetivos de la Revista de Arquitectura (Bogotá) son:

- Promover la divulgación y difusión del conocimiento generado a nivel local, nacional e internacional

- Conformar un espacio para la construcción de comunidades académicas y la discusión en torno a las secciones definidas.

- Fomentar la diversidad institucional y geográfica de los autores que participan en la publicación.

- Potenciar la discusión de experiencias e intercambios científicos entre investigadores y profesionales.

- Contribuir a la visión integral de la arquitectura, por medio de la concurrencia y articulación de las secciones mediante la publicación de artículos de calidad.

- Publicar artículos originales e inéditos que han pasado por revisión de pares, para asegurar que se cumplen las normas éticas, de calidad, validez científica, editorial e investigativa.

- Fomentar la divulgación de las investigaciones y actividades desarrolladas en la Universidad Católica de Colombia.
Palabras clave de la Revista de Arquitectura (Bogotá): arquitectura, diseño, educación arquitectónica, proyecto y construcción, urbanismo.

Idiomas de publicación: español, inglés, portugués y francés. Título abreviado: Rev. Arquit.

Titulo corto: RevArq

\section{Políticas de sección}

La revista se estructura en tres secciones correspondientes a las líneas de investigación activas y aprobadas por la institución, y dos complementarias, que presentan dinámicas propias de la Facultad de Diseño y las publicaciones relacionadas con la disciplina.

Cultura y espacio urbano. En esta sección se publican los artículos que se refieren a fenómenos sociales en relación con el espacio urbano, atendiendo aspectos de la historia, el patrimonio cultural y físico, y la estructura formal de las ciudades y el territorio.

Proyecto arquitectónico y urbano. En esta sección se presentan artículos sobre el concepto de proyecto, entendido como elemento que define y orienta las condiciones proyectuales que devienen en los hechos arquitectónicos o urbanos, y la forma como estos se convierten en un proceso de investigación y nuevo de conocimiento. También se presentan proyectos que sean resultados de investigación, los cuales se validan por medio de la ejecución y transformación en obra construida del proceso investigativo. También se contempla la publicación de investigaciones relacionadas con la pedagogía y didáctica de la arquitectura, el urbanismo y el diseño.

Tecnología, medioambiente y sostenibilidad. En esta sección se presentan artículos acerca de sistemas estructurales, materiales y procesos constructivos, medioambiente y gestión, relacionados con los entornos social-cultural, ecológico y económico.

Desde la Facultad. En esta sección se publican artículos generados en la Facultad de Diseño, relacionados con las actividades de docencia, extensión, formación en investigación o internacionalización, las cuales son reflejo de la dinámica y de las actividades realizadas por docentes, estudiantes y egresados; esta sección no puede superar el $20 \%$ del contenido.

Textos. En esta sección se publican reseñas, traducciones y memorias de eventos relacionados con las publicaciones en Arquitectura y Urbanismo.
A Frecuencia de publicación

Desde 1999 y hasta el 2015, la Revista de Arquitectura (Bogotá) publicó un volumen al año, a partir del 2016 se publicarán dos números por año en periodo anticipado, enero-junio y julio-diciembre, pero también maneja la publicación anticipada en línea de los artículos aceptados (versión Post-print del autor).

La Revista de Arquitectura (Bogotá) se divulga mediante versiones digitales (PDF, HTML, EPUB, XML) e impresascon un tiraje de 700 ejemplares, los tiempos de producción de estas versiones dependerán de los cronogramas establecidos por la editorial.

Los tiempos de recepción-revisión-aceptación pueden tardar entre seis y doce meses dependiendo del flujo editorial de cada sección y del proceso de revisión y edición adelantado.

Con el usuario y contraseña asignados, los autores pueden ingresar a la plataforma de gestión editorial y verificar el estado de revisión, edición o publicación del artículo.
A Canje

La Revista de Arquitectura (Bogotá) está interesada en establecer canje con publicaciones académicas, profesionales o científicas del área de Arquitectura y Urbanismo, como medio de reconocimiento y discusión de la producción científica en el campo de acción de la publicación.

Mecanismo

Para establecer canje por favor descargar, diligenciar y enviar el formato: RevArq FP20 Canjes

Universidad Católica de Colombia (2021,
junio-diciembre). Revista de
Arquitectura (Bogotá), 23(2),
I-132. Doi: 10.14718
ISSN: I657-0308
E-ISSN: 2357-626X
Especificaciones:
Formato: $34 \times 24 \mathrm{~cm}$
Papel: Mate II5 g
Tintas: Negro y policromía

A Contacto

Dirección postal:

Avenida Caracas N ${ }^{0} \cdot 46-72$ Universidad Católica de Colombia Bogotá D. C., Colombia Código postal: 111311

Facultad de Diseño Centro de Investigaciones (CIFAR) Sede El Claustro. Bloque "L", 4 piso Diag. 46A N ${ }^{0 .} 15 b-10$ Editor, Arq. César Eligio-Triana

Teléfonos:

+57 (1) $3277300-3277333$

Ext. $3109 ; 3112$ o 5146
Fax: +57 (1) 2858895

Correo electrónico:

revistadearquitectura@ucatolica.edu.co cifar@ucatolica.edu.co

Página WEB:

www.ucatolica.edu.co

Vínculo Revistas científicas

http://publicaciones.ucatolica.edu.co revistas-cientificas

https://revistadearquitectura.ucatolica.edu.co/ 
